\title{
ON THE NUMBER OF AUTOMORPHISMS OF A REGULAR GRAPH
}

\author{
NICHOLAS WORMALD
}

\begin{abstract}
For any connected cubic graph $G$ with $2 n$ points, the number of automorphisms of $G$ divides $3 n 2^{n}$. This is a special case of a result which is proved for connected regular graphs in general. The result is shown to be best possible for infinitely many $n$ in the cubic case.
\end{abstract}

An $r$-regular graph is a graph $G$ in which each point is of degree $r$; if $r=3$ we say $G$ is cubic. We have shown elsewhere [6] that the number of labelled connected cubic graphs with $2 n$ points is divisible by $(2 n) ! /\left(3 n 2^{n}\right)$, as are the numbers of labelled 2-connected and 3-connected cubic graphs. This was done by obtaining recurrence relations for the numbers involved. Our main concern is to verify this result directly by showing that the number of ways to label any connected cubic graph with $2 n$ points is divisible by $(2 n) ! /\left(3 n 2^{n}\right)$.

Let $s$ denote the order of the automorphism group $\Gamma(G)$ of the graph $G$. If $G$ is cubic and has $2 n$ points, it follows [5] that the number of ways to label $G$ is just $(2 n) ! / s$. Thus, our aim is fulfilled if we show that $s$ divides $3 n 2^{n}$. The following theorem is a generalisation of this result. All basic graph theoretic notation not defined here can be found in [4].

THEOREM 1. Let $G$ be a connected $r$-regular graph with p points where $r>0$. Then the number $s$ of automorphisms of $G$ divides

$$
r p \prod k^{\beta},
$$

where the product is taken over all primes $k<r-1$, and $\beta$ is defined as

$$
\sum_{k^{\alpha}<r-1}\left\lfloor\frac{p-2}{k^{\alpha}}\right\rfloor .
$$

Proof. Suppose $G$ is a connected $r$-regular graph with point set $V$ of cardinality $p$, where $r>0$. Let $U=\left\{u_{1}, u_{2}, \ldots, u_{m}\right\}$ be a subset of $V$ with $m>2$ such that the subgraph of $G$ induced by $U$ is connected. For any subset $W$ of $V$, let $n(W)$ denote the number of automorphisms of $G$ which fix each of the points in $W$. We shall show that $n(U)$ divides $\Pi k^{\gamma(p-m)}$, where

Received by the editors February 16, 1978 and, in revised form, July 10, 1978.

AMS (MOS) subject classifications (1970). Primary $05 C 25$. 
the product is taken over all primes $k \leqslant r-1$, and $\gamma(p-m)$ is defined as

$$
\sum_{k^{\alpha}<r-1}\left\llcorner\frac{p-m}{k^{\alpha}}\right\lrcorner \text {. }
$$

We use induction on $p-m$. If $m=p$ we are done because $n(U)$ is then 1 , so assume $m<p$. As $G$ is connected, there is some point $u_{i}$ in $U$ adjacent to a set $W$ of $w$ points not in $U$, for some $1<w<r-1$. The automorphisms of $G$ which fix $U$, when restricted to $W$, determine a subgroup $H$ of the symmetric group $S_{w}$ on $W$, and it is clear that the order of $H$ is just $n(U) / n(U \cup W)$. It follows that $n(U)$ divides $w ! n(U \cup W)$. By induction $n(U \cup W)$ divides $\Pi k^{\gamma(p-m-w)}$, where the product is again taken over all primes $k<r-1$. But

$$
j=\left(\prod k^{\gamma(p-m)}\right) /\left(\prod k^{\gamma(p-m-w)}\right)
$$

is an integer, and for each prime $k \leqslant w$ there are at least $\gamma(w)$ factors of $k$ in $j$ because $w<r-1$. Consequently, $w$ ! divides $j$ and thus $n(U)$ divides $\Pi k^{\gamma(p-m)}$.

In particular, if $U=\left\{u_{1}, u_{2}\right\}$, we have $n(U)$ dividing $\Pi k^{\beta}$. Now consider the action of $\Gamma(G)$ on the ordered pairs $\left(v_{1}, v_{2}\right)$ of adjacent points of $G$. Let the orbits of these ordered pairs be $O_{1}, O_{2}, \ldots, O_{i}$. If we take $\left(u_{1}, u_{2}\right) \in O_{i}$, it is clear that $s=\left|O_{i}\right| n(U)$. Thus $s$ divides $\left|O_{i}\right| \Pi k^{\beta}$ for each $i$. It follows that $s$ divides the sum of these numbers, which is just $r p \Pi k^{\beta}$.

COROLLARY 1. The number of automorphisms of a connected cubic graph with $2 n$ points divides $3 n 2^{n}$.

COROLLARY 2. The number of automorphisms of a connected 4-regular graph with $p$ points divides $2^{\llcorner(p+2) / 2\lrcorner} 3^{\llcorner(p-2) / 3} \frac{1}{p}$.

For any positive $r$ and $p$, Theorem 1 provides a number which is a common multiple of the numbers of automorphisms of the connected $r$-regular graphs with $p$ points. This is not always the least common multiple (for example, with $r=3$ and $2 n=p=8$ the least common multiple is $3 n 2^{n-2}$ ) so it is clear that the result of Theorem 1 can be improved. However, we shall demonstrate that for $r=3$, this least common multiple is in fact $3 n 2^{n}$ for an infinite number of $p=2 n$.

THEOREM 2. Let $j$ be a positive integer, and $n=3 \cdot 2^{j}-1$. Then the least common multiple of the numbers of automorphisms of the connected cubic graphs with $2 n$ points is $3 n 2^{n}$.

Proof. Let $M_{n}$ denote the least common multiple of the numbers of automorphisms of the connected cubic graphs with $2 n$ points. By Corollary 1 , we have $M_{n} \mid 3 n 2^{n}$. To show the converse, we first note that since $n=3 \cdot 2^{j}-$ 1 , it follows that $n$ is prime to 2 and 3. Thus, it is enough to show $3\left|M_{n}, n\right| M_{n}$ and $2^{n} \mid M_{n}$. 
We first show $3 \mid M_{n}$. If $n=5$, this is true since the automorphism group of the Petersen graph has order 120 , so that $3 \mid M_{5}$. If $n>5$ we have $j>2$, so that $n=3 \cdot 2^{j}-1>11$. It follows that $(2 n-4) / 3$ is an even integer greater than 4 , and thus there is some connected cubic graph $G_{1}$ with precisely $(2 n-4) / 3$ points. Introduce a new point, $u$ say, into the middle of one of the lines of $G_{1}$ and call the resulting graph $G_{1}^{\prime}$. Then let $G_{2}^{\prime}$ and $G_{3}^{\prime}$ be two other copies of $G_{1}^{\prime}$, introduce a new point $v$, and add lines from $v$ to the point $u$ and the points of $G_{2}^{\prime}$ and $G_{3}^{\prime}$ corresponding to $u$. Call the resulting graph $G$. It is clear that $G$ is connected, cubic and has $2 n$ points. Furthermore, there is clearly an automorphism $\alpha$ of $G$ which fixes $v$ and maps the points of $G_{1}^{\prime}$ to those of $G_{2}^{\prime}$, those of $G_{2}^{\prime}$ to those of $G_{3}^{\prime}$, and those of $G_{3}^{\prime}$ to those of $G_{1}^{\prime}$, such that $\alpha^{3}$ is the identity automorphism of $G$. Thus 3 divides $s$ and hence $3 \mid M_{n}$.

To show that $n \mid M_{n}$ it is enough to find a connected cubic graph on $2 n$ points which has an automorphism permuting the points in a cycle of length $n$. The graph $C_{n} \times K_{2}$ has this property.
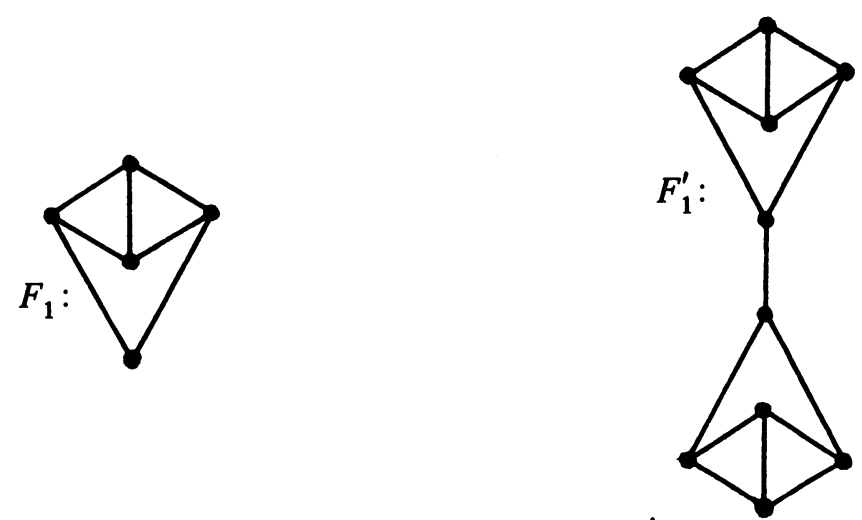

FIGURE. The graphs $F_{1}$ and $F_{1}^{\prime}$.

It remains to show that $2^{n} \mid M_{n}$. Let $F_{1}$ be the graph shown in the left side of the Figure. Define $F_{j}$ inductively to be the graph formed by taking two copies of $F_{j-1}$, introducing a new point $v$ and adding lines from $v$ to the points of degree 2 in the two copies of $F_{j-1}$. Also define $F_{j}^{\prime}$ to be the graph obtained by taking two copies of $F_{j}$ and adding a line between the points of degree 2 . Thus, for example, the graph shown in the right side of the Figure is $F_{1}^{\prime}$. It is clear that $F_{j}^{\prime}$ is a connected cubic graph with $2 n$ points, and it is not hard to see that the automorphism group of $F_{j}^{\prime}$ in fact has cardinality $2^{n}$. Thus $2^{n} \mid M_{n}$ as required.

Theorem 1 can also be viewed from another angle: it supplies a lower bound on the number of points in an $r$-regular graph with an automorphism group of a given size. Frucht [3] sought to show that for each finite group $X$ there is a cubic graph $G$ such that $\Gamma(G) \cong X$, and attempted to minimise the size of such a graph $G$. Babai [1] approached this problem from the other direction and deduced the following lower bound for the size of $G$. Suppose $X$ 
has order $t$ with $(t, 6)=1$, is minimally generated by $f$ generators, and is indecomposable with respect to the direct product. Then the minimum order of a cubic graph $G$ with $\Gamma(G) \cong X$ is $2 j t$ for some integer $j \geqslant f-1$. On the other hand, by Corollary 1 we know that for any finite group $X$ of order $t$, if $2 n$ is the order of a cubic graph $G$ with $\Gamma(G) \cong X$ then $3 n 2^{n} \geqslant t$. The latter result appears to be far from best possible when $t$ is large, or even for most groups $X$ when $t$ is small.

Finally, we wish to point out that arguments related to those of this paper, but applied somewhat differently, can be found in [2].

\section{REFERENCES}

1. L. Babai, Some applications of graph contractions, J. Graph Theory 1 (1977), 125-130.

2. L. Babai and L. Lovasz, Permutation groups and almost regular graphs, Studia Sci. Math. Hungar. 8 (1973), 141-150.

3. R. Frucht, Graphs of degree three with a given abstract group, Canad. J. Math. 1 (1949), 365-378.

4. F. Harary, Graph theory, Addison-Wesley, Reading, Mass., 1969.

5. F. Harary, E. M. Palmer and R. C. Read, The number of ways to label a structure, Psychometrika 32 (1967), 155-156.

6. N. Wormald, Enumeration of labelled graphs II: Cubic graphs with a given connectivity, J. London Math. Soc. (to appear).

Department of Mathematics, Universtry of Newcastue, New South Wales, 2308, Australla 